\title{
Co-existence of Sarcoidosis of Cervical Lymph Nodes and Breast Carcinoma - a rare case report and review of literature
}

\author{
Dr Avinash $\mathbf{M J}^{1}$ \\ 'Surgeon, Raj Mahal Vilas Hospital, 138, ACES Layout, Sanjaynagar, Bangalore-560094 \\ DOI - 10.46319/RJMAHS.2019.v02i01.010
}

\begin{abstract}
Sarcoidosis is a chronic disease affecting multiple systems and is characterized by non-caseating granulomas. It is of unknown etiology. In some cases it is very difficult to differentially diagnose sarcoidosis from malignancy just by clinical and radiological features in spite of modern imaging modalities like PET-CT Scan. Its association with solid organ malignancy is rare. The only accurate way to demonstrate and differentiate sarcoidosis from malignancy is histopathological examination. We report a rare case of Sarcoidosis affecting right sided cervical lymphnodes coexisting in a patient with left sided breast carcinoma.
\end{abstract}

Keywords: Breast cancer, metastasis, sarcoidosis, lymphadenopathy, noncaseating granuloma.

\section{Introduction}

Sarcoidosis whose etiology is unknown can affect different organs and systems. Sarcoidosis is a chronic inflammatory disorder. Sarcoidosis is known to imitate breast cancer, which makes the diagnosis difficult. ${ }^{[1]}$ Sarcoidosis can present with following symptoms: cough, difficulty in breathing, febrile arthritis, erythema nodosum, parotitis and uveitis. About half of the patients are symptomless during diagnosis. Sarcoidosis usually affects hilar and mediastinal lymph nodes. ${ }^{[2]}$ Sarcoidosis is characterized histopathologically by noncaseating epitheloid cell granulomas ${ }^{[3]}$ Even though the relation between sarcoidosis and malignancy has been extensively studied, their correlation remains unclear. ${ }^{[2]}$ Here in this case report we describe a case of Sarcoidosis affecting right lower deep cervical lymphnodes suspected to be metastatic in an elderly lady who suffered from Invasive ductal carcinoma of left breast (pT2 N1a Grade 2).Case report: A 70 year old lady came to the surgical OPD with history of progressively increasing lump in the left breast noted by her 2 months prior to consultation. She had no other significant complaints. Clinical examination revealed a hard irregular nontender lump in the lower quadrant of the left breast associated with axillary lymphadenopathy. Her mammogram revealed features of a suspicious breast mass in lower pole of left breast. She underwent an uneventful Left sided breast conservative surgery along with left sided axillary block dissection. Her histopathological examination report showed features of Grade 2 Invasive ductal carcinoma. One out of 9 lymphnodes isolated was positive for malignancy. The resected margins, NAC and resected skin was free from tumour. She was evaluated postoperatively by medical oncologist with PET-CT scan that revealed metabolically active Right side deep cervical and mediastinal lymphnodes. She underwent right sided medial deep cervical lymphnode excision biopsy. Totally 13 lymphnodes were excised; 8 nodes more than $1 \mathrm{~cm}$ in diameter, largest $3.7 \mathrm{~cm}, 5$ nodes lesser than 1 $\mathrm{cm}$ in diameter (Figure 1). Microscopically all lymph node structure was effaced with presence of non caseating (hard) granulomas (Figure 2). These hard granulomas showed multiple giant cells, Schaumann bodies-laminated congretion of calcium and protein (Figure 3), Asteroid bodies (Figure 4) extracellular collagen - collagenous fibrous tissue. No evidence of metastatic tumor cells were found in any of the 13 lymph nodes examined. The postoperative diagnosis was left breast carcinoma (pT2N1aM0) with sarcoidosis of right cervical lymphnodes.

\section{Discussion:}

Breast cancer and sarcoidosis affects female population

Address for Correspondence:

Dr. Avinash MJ, DNB (Gen.Surg), MRCS ED

41, 9th Main, 1st Cross, NTI Layout, Vidyaranyapura,

Bangalore-560097

E-mail: dravinashmelkotermv@gmail.com 


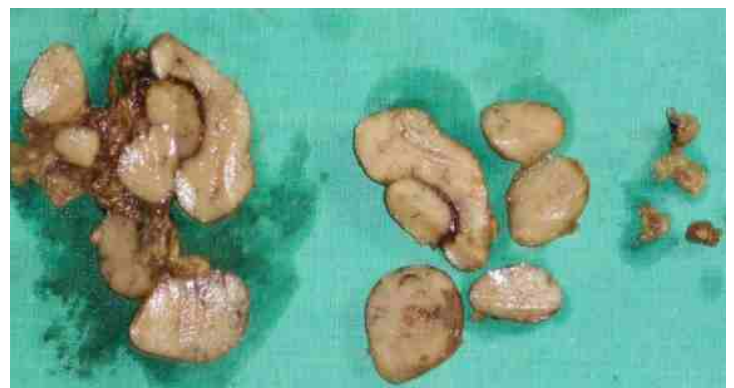

Fig 1. Excised right deep cervical lymphnodes of varying sizes.

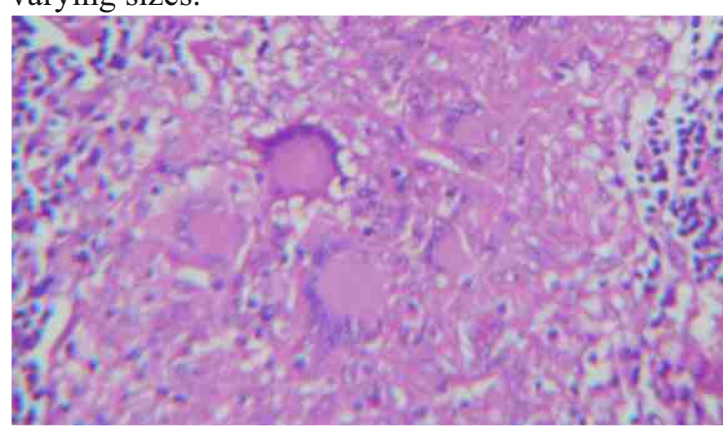

Fig 2. Hard granuloma without caseation with Langhans type giant cells. H\&E X 200.

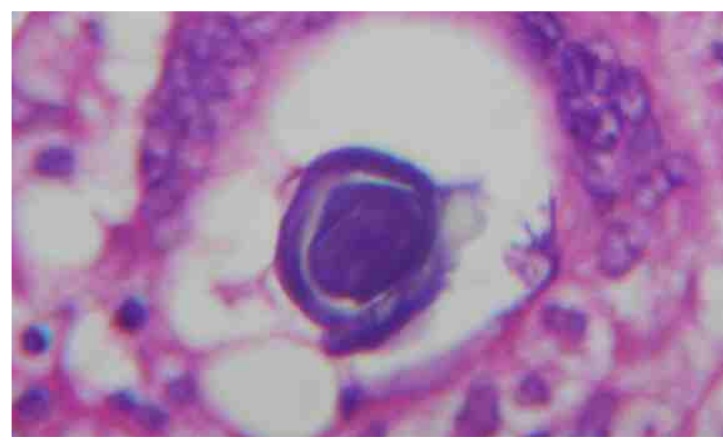

Figure 3. Schaumann body. H\&E X 400.

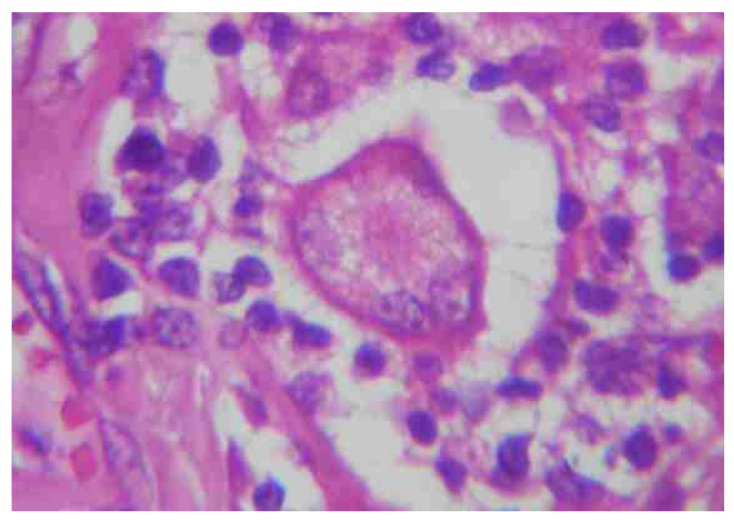

Figure 4. Asteroid body. H\&E X 400.

more than the male population..$^{[4]}$ The association between sarcoidosis and carcinogenesis is not proven. However there are many studies in the literature to mention about such association. First study to find such link was carried out by Brincker and Wilbek. They studied 2544 sarcoidosis cases. In patients with sarcoidosis than those in the general population, the incidence of lymphomas and lung cancer were 11 and 3 folds higher. The incidence of breast cancer is more among patients with sarcoidosis. Hunt et al. has described 21 sarcoidosis cases that developed after primary malignancies. Among 30 patients with sarcoidosis and malignancies Butt et al. has reported 10 cases of breast cancer. As reported by Blank et al, cervical cancer, Breast cancer and B-cell lymphomas were the most common malignancies in patients diagnosed with sarcoidosis. ${ }^{[2]}$

Sarcoid lesions may imitate recurrence or metastasis in cancer patients. Biopsy followed by histopathology examination should be carried out and diagnosis should be confirmed. Presence of active cancer or its therapy may lead to altered immunologic milieu which inturn has been proposed to cause recurrence of sarcoidosis. $^{[5]}$

Many possible sequential associations exist between breast cancer and sarcoidosis. In our case the diagnosis of sarcoidosis was made immediately after breast cancer diagnosis and surgery.

Sarcoidosis commonly affects lungs. It is often associated with hilar or mediastinal lymphadenopathy. One of the most common extrapulmonary presentations include peripheral lymph node involvement. The peripheral lymphnode involvement has been reported in about $8-15 \%$ of patients. Clinical examination findings may remain same in both sarcoidosis and breast masses. Imaging alone will not be helpful in differentiating breast involvement in sarcoidosis from breast malignancy. ${ }^{[1]}$

Positron emission tomography-Computerised Tomography scan (FDG-PET-CT scan) is an advanced imaging tool to assess the extent of malignant disease. The sensitivity and specificity of PET-CT scan as a diagnostic tool to identify malignant lesions is between $47-100 \%$ and $86-100 \%$ respectively. ${ }^{[6]}$ However the limitation with FDG$\mathrm{PET} / \mathrm{CT}$ scan in cases where patient has both malignancy and granulomatous disease simultaneously is that both granulomatous tissue and malignant tissue will take-up the flurodeoxyglucose. Studies have shown that the patients with malignancies have significantly higher standardized 
uptake value (SUV) than with benign conditions. ${ }^{[7]}$ However the patients with granulomatous process, have maximal SUV similar to patients with malignant diseases. Hence, FDG-PET/CT scan as additional diagnostic tool helps to assess the extent of disease spread. FDG-PET/CT scan will not be helpful in differentiating between malignant and granulomatous disorders. With coexisting pathologies this imaging tool may lead to diagnostic difficulties and misunderstandings. Only histopathological confirmation describes the nature of the disease accurately. ${ }^{[2]}$

The usefulness of PET Scan as an imaging modality in diagnosing sarcoidosis may be improved in the future by usage of other tracers. A combination of $18 \mathrm{~F}-\mathrm{FDG}$ and fluorine-18-methyltyrosine (18F-FMT) PET scanning did differentiate sarcoidosis from malignancy as described in a study consisting of 24 cases of sarcoid and 17 cases of lung cancer. Sarcoid lesions were positive on 18F-FDG PET, but negative on 18F-FMT PET but both scans are positive in cancer patients. ${ }^{[8]}$

To overcome the current diagnostic difficulty further cell subset analysis in these pathologies may reveal immunological distinct cell populations as useful markers to help differentiate sarcoidosis, cancer and the coexistence of these two. ${ }^{[6]}$

To date about 53 cases of association of sarcoidosis and breast carcinoma has been reported in English literature. As per our best knowledge no case has been reported in Indian literature. Hence we are reporting this rare case.

In our case patient presented with breast lump that was proved to be malignant and subsequently was suspected to have metastasis by PET-CT scan in cervical lymphnodes. However histopathological examination of the cervical lymph nodes revealed sarcoidosis features. This rare case report demonstrates the value of differential diagnosis of peripheral lymphadenopathy in patients with malignancy. Also presence of other benign pathologies as demonstrated by histopathological examination in such cases will rule out presence of malignancy leading to change in postoperative management of the patient.

Conclusion: Noncancerous pathologies may coexist with malignancy in any patient. Hence it becomes mandatory to obtain a proper tissue diagnosis to prove the metastatic nature of the lesion before starting them on cancer related therapy.

\section{Financial Support and sponsorship: Nil Conflicts of interest: Nil}

Acknowledgement: I acknowledge with thanks Dr.C.S. Belagavi, Consultant Pathologist, RMV Hospital, Bangalore for the valuable guidance.

\section{References:}

1. Jacob Chen, Robert Carter III, Daniel Maoz, Ana Tobar, Eran Sharon, Franklin Greif. Breast Cancer and Sarcoidosis: Case Series and Review of the Literature. Novel Insights from Clinical Practice. Breast Care 2015;10:137-140. DOI: 10.1159/000381324. Available from: www.karger.com/brc.

2. Kochoyan T, Akhmedov M, Shabanov A, Terekhov I. Sarcoidosis imitating breast cancer metastasis: a case report and literature review. Cancer Biol Med. 2016; 13: 396-8. doi: 10.20892/j.issn.2095-3941.2016.0038. Available from: www.cancerbiomed.org

3. Jacqueline Deen, Nick Mellick, and Laura Wheller. Concurrent Diagnoses of Cutaneous Sarcoidosis and Recurrent Metastatic Breast Cancer: More than a Coincidental Occurrence? Case Reports in Dermatological Medicine Volume 2018, Article ID 2812439 , 6 pages. Available from: https://doi.org/10.1155/2018/2812439.

4. M. D. Schweitzer, O. Salamo, G. Holt, E. Donna, and M. Mirsaeidi, "Sarcoidosis onset after breast cancer; a potential association," European Journal of Internal Medicine, vol. 44, pp. e11-e12, 2017.

5. Myint ZW, Chow RD. Sarcoidosis mimicking metastatic thyroid cancer following radioactive iodine therapy. J Community Hosp Intern Med Perspect. 2015 Feb 3;5(1):26360. doi: 10.3402/jchimp.v5.26360. eCollection 2015.

6. Christoph Spiekermann, Meike Kuhlencord, Sebastian Huss, Claudia Rudack and Daniel weiss. Coexistence of sarcoidosis and metastatic lesions: A diagnostic and therapeutic dilemma (Review). Oncology Letters 14: 7643-7652, 2017.

7. Karam M, Roberts-Klein S, Shet N, Chang J, Feustel P. Bilateral hilar foci on 18F-FDG PET scan in patients without lung cancer: variables associated with benign and malignant etiology. J Nucl Med. 2008; 49: 1429-36.

8. Kaira K, Oriuchi N, Otani Y, Yanagitani N, Sunaga N, Hisada T, et al. Diagnostic usefulness of fluorine-18alpha-methyltyrosine positron emission tomography in combination with $18 \mathrm{~F}$-fluorodeoxyglucose in sarcoidosis patients. Chest 2007; 131:1019. 